\title{
Refinement of the crystal structure of barium tetrafluoridobromate(III) from single-crystal X-ray diffraction data
}

\author{
Sergei I. Ivlev and Florian Kraus* \\ Philipps-Universitaet Marburg, Fachbereich Chemie, Hans-Meerwein-Str. 4, 35032 Marburg, Germany \\ Correspondence email: f.kraus@uni-marburg.de
}

\section{Abstract}

Single-crystals of barium tetrafluoridobromate(III), $\mathrm{Ba}\left[\mathrm{BrF}_{4}\right]_{2}$, were obtained in form of tiny blocks. Crystal structure refinement of $\mathrm{Ba}\left[\mathrm{BrF}_{4}\right]_{2}$ from single-crystal X-ray diffraction data confirmed the previous model on basis of powder data [Ivlev et al. (2014). Eur. J. Inorg. Chem., pp 6261-6267], but with all atoms refined with anisotropic displacement parameters. The crystal structure consists of two symmetry-independent barium cations that are coordinated by twelve fluorine atoms each, forming edge-sharing polyhedra, and an almost square planar $\left[\mathrm{BrF}_{4}\right]^{-}$anion. The compound crystallizes in the $\mathrm{Ba}\left[\mathrm{AuF}_{4}\right]_{2}$ structure type.

\section{Structure description}

The first synthesis of $\mathrm{Ba}\left[\mathrm{BrF}_{4}\right]_{2}$ was perfomed by Sharpe \& Emeléus (1948) by treating anhydrous barium chloride or barium fluoride with bromine trifluoride. The product was, however, only characterized by means of a quantitative elemental analysis. Thermal properties of $\mathrm{Ba}\left[\mathrm{BrF}_{4}\right]_{2}$ were later studied later by Kiselev and co-workers, who investigated the thermal decomposition of $\mathrm{Ba}\left[\mathrm{BrF}_{4}\right]_{2}$ to yield barium fluoride (Kiselev et al., 1987). To the best of our knowledge, our report on the crystal structure of $\mathrm{Ba}\left[\mathrm{BrF}_{4}\right]_{2}$ determined from $\mathrm{X}$-ray and neutron powder diffraction data at $300 \mathrm{~K}$ was the first structural investigation of the title compound (Ivlev et al., 2014). We showed that $\mathrm{Ba}[\mathrm{BrF}]_{2}$ crystallizes in the space group $I \overline{4}$ and adopts the $\mathrm{Ba}\left[\mathrm{AuF}_{4}\right]_{2}$ structure type. Here we present our results on the re-refinement of the crystal structure of $\mathrm{Ba}\left[\mathrm{BrF}_{4}\right]_{2}$ from single-crystal X-ray diffraction data at $100 \mathrm{~K}$.

As expected, the tnit cell parameters of the single-crystal study at $100 \mathrm{~K}$ (Table 1) are smaller than those determined during the powder study at $300 \mathrm{~K}, a=9.65081$ (11), $c=8.03453$ (13) $\AA, V=748.32$ (2) $\AA^{3}$ (Ivlev et al., 2014). The crystal structure contains two symmetry-independent $\mathrm{Ba}^{2+}$ cations on special Wyckoff position $2 a$ (site symmetry $\overline{4}$..) and $2 d$ (4...), respectively. Each Ba site is coordinated by twelve $\mathrm{F}$ atoms forming edge-sharing polyhedra. The Ba $\cdots \mathrm{F}$ distances

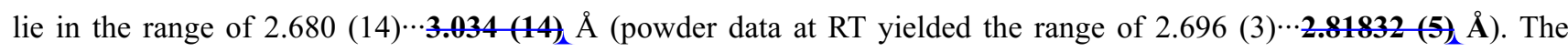
bromine atom occupies the general Wyckoff position $8 g$ and is coordinated by four fluorine atoms also located on general positions in an almost square-planar shape. The resulting $\mathrm{Br}-\mathrm{F}$ bond lengths are 1.829 (13), 1.861 (12), 1.934 (13), and 1.935 (13) $\AA$, which is comparable with our previous model on basis of powder data (cf:: 1.800 (4), 1.856 (4), 1.902 (4), 1.935 (2) $\AA$ ). The two longer $\mathrm{Br}-\mathrm{F}$ bond lengths correspond to the $\mathrm{F}$ atoms coordinating two barium cations each. The two other fluorine atoms coordinate only to one barium cation each and thus have shorther $\mathrm{Br}-\mathrm{F}$ bond lengths. The $\mathrm{F}-$ $\mathrm{Br}-\mathrm{F}$ cis-angles equal 84.9 (6), $89.6(6), 92.6(6)$, and $92.9(6)^{\circ}$, which corresponds to the previously published results: $85.14(16), 90.02(13), 91.80(15), 93.04(18)^{\circ}$. Fig. 1 shows the closest contacts between one $\left[\mathrm{BrF}_{4}\right]^{-}$anion and its surrounding $\mathrm{Ba}^{2+}$ cations, and Fig. 2 shows the packing of the cations and anions in the crystal structure.

\section{Synthesis and crystallization}

Tiny crystals of barium tetrafluoridobromate(III) were obtained by direct reaction of bromine trifluoride with barium fluoride in a closed Teflon vessel. In contrast to $\mathrm{Rb}\left[\mathrm{BrF}_{4}\right]$ (Malin et al., 2019) and $\mathrm{Cs}\left[\mathrm{BrF}_{4}\right]$ (Malin et al., 2020), it was not possible to improve the crystal quality by melting and recrystallization since $\mathrm{Ba}[\mathrm{BrF}]_{2}$ decomposes before reaching 
its melting point.

\section{Refinement details}

Details of data collection and structure refinement are given in Table 1. Due to very small size of the crystals, we had to employ a diffractometer with a $\mathrm{Cu}$ source to improve the reflection intensities at the cost of a more complicated absorption correction.

\section{Table 1}

\section{Experimental details}

\section{Crystal data}

Chemical formula

$M_{\mathrm{r}}$

Crystal system, space group

Temperature $(\mathrm{K})$

$a, c(\AA)$

$V\left(\AA^{3}\right)$

Z

Radiation type

$\mu\left(\mathrm{mm}^{-1}\right)$

Crystal size (mm)

Data collection

Diffractometer

Absorption correction

$T_{\min }, T_{\max }$

No. of measured, independent and observed $[I>2 \sigma(I)]$ reflections

$R_{\text {int }}$

$(\sin \theta / \lambda)_{\max }\left(\AA^{-1}\right)$

Refinement

$R\left[F^{2}>2 \sigma\left(F^{2}\right)\right], w R\left(F^{2}\right), S$

No. of reflections

No. of parameters

$\Delta \rho_{\max }, \Delta \rho_{\min }\left(\mathrm{e} \AA^{-3}\right)$

Absolute structure

Absolute structure parameter

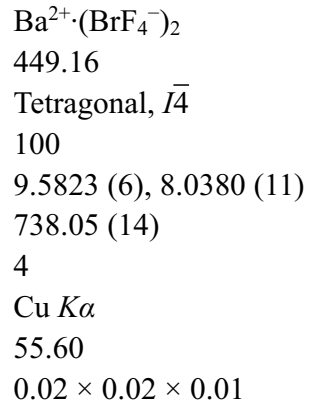

Stoe Stadivari

Multi-scan

(X-AREA (Stoe, 2020) based on Koziskova et al., (2016))

$0.068,0.362$

2012, 746, 688

0.038

0.637

$0.049,0.127,1.08$

746

50

$1.84,-1.05$

Flack x determined using 266 quotients [(I+)-(I-)]/[(I+)+(I-)] (Parsons et al., 2013). -0.012 (17)

Computer programs: X-AREA (Stoe, 2020), X-AREA (Stoe, 2020), SHELXT (Sheldrick, 2015a), SHELXL (Sheldrick, 2015b), DIAMOND (Brandenburg, 2020), publCIF (Westrip, 2015).

\section{Acknowledgements}

We thank the Deutsche Forschungsgemeinschaft for generous funding.

\section{Funding information}




\section{References}

Brandenburg, K. (2020). DIAMOND. Crystal Impact GbR, Bonn, Germany.

Ivlev, S., Sobolev, V., Hoelzel, M., Karttunen, A. J., Müller, T., Gerin, I., Ostvald, R. \& Kraus, F. (2014). Eur. J. Inorg. Chem., pp. 6261-6267.

Kiselev, N. I., Lapshin, O. N., Sadikova, A. T., Sukhoverkhov, V. F. \& Churbanov, M. F. (1987). Visok. Veschestva, 3, $178-182$.

Koziskova, J., Hahn, F., Richter, J. \& Kozisek, J. (2016). Acta Chim. Slov. 9, 136-140.

Malin, A. V., Ivlev, S. I., Ostvald, R. V. \& Kraus, F. (2019). IUCrData, 4, x191595.

Malin, A. V., Ivlev, S. I., Ostvald, R. V. \& Kraus, F. (2020). IUCrData, 5, x200114.

Parsons, S., Flack, H. D. \& Wagner, T. (2013). Acta Cryst. B69, 249-259.

Sharpe, A. G. \& Emeléus, H. J. (1948). J. Chem. Soc., pp. 2135-2138.

Sheldrick, G. M. (2015a). Acta Cryst. A71, 3-8.

Sheldrick, G. M. (2015b). Acta Cryst. C71, 3-8.

Stoe (2020). $X$-AREA. Stoe \& Cie GmbH, Darmstadt, Germany.

Westrip, S. P. (2010). J. Appl. Cryst. 43, 920-925.

Figure 1

Caption 1 (including probabilty level and-symmetry codes), Caption 2 


\section{full crystallographic data}

\section{Refinement of the crystal structure of barium tetrafluoridobromate(III) from single-crystal $X$-ray diffraction data}

(I)

\section{Crystal data}

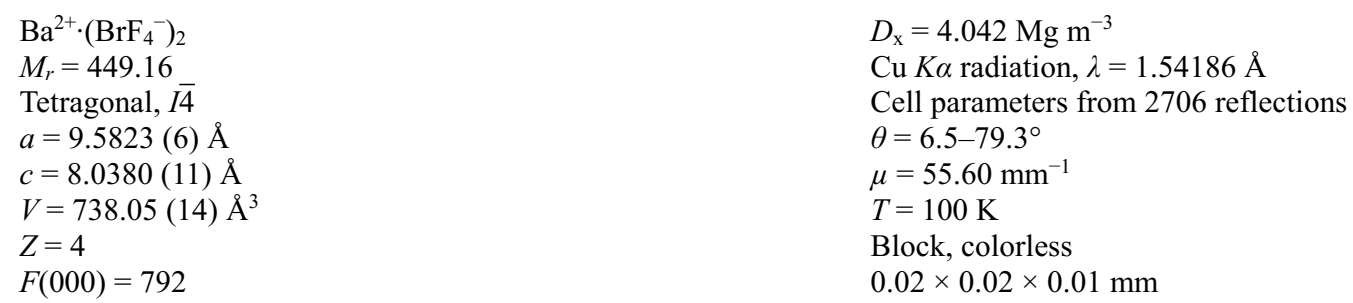

$0.02 \times 0.02 \times 0.01 \mathrm{~mm}$

\section{Data collection}

Stoe Stadivari diffractometer

Radiation source: GeniX 3D HF Cu

Graded multilayer mirror monochromator

Detector resolution: 5.81 pixels $\mathrm{mm}^{-1}$

rotation method, $\omega$ scans

Absorption correction: multi-scan

(X-AREA (Stoe, 2020) based on Koziskova et al., (2016))

$$
\begin{aligned}
& T_{\min }=0.068, T_{\max }=0.362 \\
& 2012 \text { measured reflections } \\
& 746 \text { independent reflections } \\
& 688 \text { reflections with } I>2 \sigma(I) \\
& R_{\text {int }}=0.038 \\
& \theta_{\max }=79.1^{\circ}, \theta_{\min }=6.5^{\circ} \\
& h=-11 \rightarrow 12^{\circ} \\
& k=-10 \rightarrow 6 \\
& l=-10 \rightarrow 9
\end{aligned}
$$

\section{Refinement}

Refinement on $F^{2}$

Least-squares matrix: full

$R\left[F^{2}>2 \sigma\left(F^{2}\right)\right]=0.049$

$w R\left(F^{2}\right)=0.127$

$S=1.08$

746 reflections

50 parameters

0 restraints

Primary atom site location: structure-invariant direct methods

$w=1 /\left[\sigma^{2}\left(F_{\mathrm{o}}{ }^{2}\right)+(0.091 P)^{2}+5.3796 P\right]$

where $P=\left(F_{\mathrm{o}}^{2}+2 F_{\mathrm{c}}^{2}\right) / 3$

$(\Delta / \sigma)_{\max }<0.001$

$\Delta \rho_{\max }=1.84 \mathrm{e} \AA^{-3}$

$\Delta \rho_{\min }=-1.05$ e $\AA^{-3}$

Absolute structure: Flack x determined using 266 quotients [(I+)-(I-)]/[(I+)+(I-)] (Parsons et al., 2013).

Absolute structure parameter: -0.012 (17)

\section{Special details}

Geometry. All e.s.d.'s (except the e.s.d. in the dihedral angle between two 1.s. planes) are estimated using the full covariance matrix. The cell e.s.d.'s are taken into account individually in the estimation of e.s.d.'s in distances, angles and torsion angles; correlations between e.s.d.'s in cell parameters are only used when they are defined by crystal symmetry. An approximate (isotropic) treatment of cell e.s.d.'s is used for estimating e.s.d.'s involving l.s. planes.

Fractional atomic coordinates and isotropic or equivalent isotropic displacement parameters $\left(\AA^{2}\right)$

\begin{tabular}{lllll}
\hline & $x$ & $y$ & $z$ & $U_{\text {iso }} * / U_{\text {eq }}$ \\
\hline Ba1 & 0.500000 & 0.500000 & 0.500000 & $0.0243(6)$ \\
$\mathrm{Br} 1$ & $0.77576(19)$ & $0.84257(18)$ & $0.6325(2)$ & $0.0238(5)$ \\
$\mathrm{F} 1$ & $0.7435(16)$ & $0.6574(14)$ & $0.5444(17)$ & $0.041(3)$
\end{tabular}




\begin{tabular}{|c|c|c|c|c|}
\hline $\mathrm{Ba} 2$ & 0.500000 & 1.000000 & 0.250000 & $0.0293(6)$ \\
\hline F2 & $0.6192(14)$ & $0.9136(15)$ & $0.5334(19)$ & $0.041(3)$ \\
\hline F3 & $0.8236(13)$ & $1.0143(13)$ & $0.7242(17)$ & $0.036(3)$ \\
\hline F4 & $0.9413(13)$ & $0.7748(13)$ & $0.744(2)$ & $0.040(3)$ \\
\hline
\end{tabular}$$
1
$$

Atomic displacement parameters $\left(\AA^{2}\right)$

\begin{tabular}{lllllll}
\hline & $U^{11}$ & $U^{22}$ & $U^{33}$ & $U^{12}$ & $U^{13}$ & $U^{23}$ \\
\hline $\mathrm{Ba} 1$ & $0.0219(7)$ & $0.0219(7)$ & $0.0291(12)$ & 0.000 & 0.000 & 0.000 \\
$\mathrm{Br} 1$ & $0.0251(10)$ & $0.0197(9)$ & $0.0267(9)$ & $-0.0021(7)$ & $-0.0004(8)$ & $0.0000(7)$ \\
$\mathrm{F} 1$ & $0.050(8)$ & $0.030(7)$ & $0.043(7)$ & $-0.013(6)$ & $0.008(6)$ & $-0.008(5)$ \\
$\mathrm{Ba} 2$ & $0.0257(8)$ & $0.0257(8)$ & $0.0364(13)$ & 0.000 & 0.000 & 0.000 \\
$\mathrm{~F} 2$ & $0.033(7)$ & $0.042(7)$ & $0.048(8)$ & $0.004(6)$ & $-0.009(6)$ & $-0.004(6)$ \\
$\mathrm{F} 3$ & $0.042(7)$ & $0.027(6)$ & $0.038(8)$ & $-0.001(5)$ & $-0.006(6)$ & $-0.003(5)$ \\
$\mathrm{F} 4$ & $0.034(7)$ & $0.029(6)$ & $0.057(8)$ & $0.003(6)$ & $-0.017(7)$ & $-0.006(6)$ \\
\hline
\end{tabular}

Geometric parameters $\left(\AA,{ }^{\circ}\right)$

\begin{tabular}{|c|c|c|c|}
\hline $\mathrm{Ba} 1-\mathrm{F} 3^{\mathrm{i}}$ & $2.791(13)$ & $\mathrm{Br} 1-\mathrm{F} 2$ & $1.829(13)$ \\
\hline $\mathrm{Ba} 1-\mathrm{F} 3^{\mathrm{ii}}$ & $2.791(13)$ & $\mathrm{Br} 1-\mathrm{F} 3$ & $1.861(12)$ \\
\hline $\mathrm{Ba} 1-\mathrm{F} 3^{\mathrm{iii}}$ & $2.791(13)$ & $\mathrm{Br} 1-\mathrm{F} 4$ & $1.934(13)$ \\
\hline $\mathrm{Ba} 1-\mathrm{F} 3^{\text {iv }}$ & $2.791(13)$ & $\mathrm{Br} 1-\mathrm{F} 1$ & $1.935(13)$ \\
\hline $\mathrm{Ba} 1-\mathrm{F} 1^{\mathrm{v}}$ & $2.801(14)$ & $\mathrm{Ba} 2-\mathrm{F}^{\text {viii }}$ & $2.680(14)$ \\
\hline $\mathrm{Ba} 1-\mathrm{F} 1^{\mathrm{vi}}$ & $2.801(14)$ & $\mathrm{Ba} 2-\mathrm{F} 2^{\mathrm{ix}}$ & $2.680(14)$ \\
\hline $\mathrm{Ba} 1-\mathrm{F} 1^{\mathrm{vii}}$ & $2.801(14)$ & $\mathrm{Ba} 2-\mathrm{F}^{\mathrm{x}}$ & $2.680(14)$ \\
\hline $\mathrm{Ba} 1-\mathrm{F} 1$ & $2.801(14)$ & $\mathrm{Ba} 2-\mathrm{F} 2$ & $2.680(14)$ \\
\hline $\mathrm{Ba} 1-\mathrm{F}^{\mathrm{ii}}$ & $3.034(14)$ & $\mathrm{Ba} 2-\mathrm{F}^{\mathrm{xi}}$ & $2.693(12)$ \\
\hline $\mathrm{Ba} 1-\mathrm{F} 4^{\mathrm{iv}}$ & $3.034(14)$ & $\mathrm{Ba} 2-\mathrm{F} 4^{\mathrm{vii}}$ & $2.693(12)$ \\
\hline $\mathrm{Ba} 1-\mathrm{F} 4^{\mathrm{iii}}$ & $3.034(14)$ & $\mathrm{Ba} 2-\mathrm{F} 4^{\mathrm{iii}}$ & $2.693(12)$ \\
\hline $\mathrm{Ba} 1-\mathrm{F}^{\mathrm{i}}$ & $3.034(14)$ & $\mathrm{Ba} 2-\mathrm{F} 4^{\mathrm{xii}}$ & $2.693(12)$ \\
\hline $\mathrm{F} 3^{\mathrm{i}}-\mathrm{Ba} 1-\mathrm{F}^{\mathrm{ii}}$ & $129.1(3)$ & $\mathrm{F} 4^{\mathrm{ii}}-\mathrm{Ba} 1-\mathrm{F} 4^{\mathrm{iii}}$ & $94.6(6)$ \\
\hline 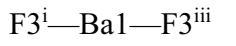 & $129.1(3)$ & $\mathrm{F} 4^{\mathrm{iv}}-\mathrm{Ba} 1-\mathrm{F} 4^{\mathrm{iii}}$ & $117.4(3)$ \\
\hline $\mathrm{F} 3^{\mathrm{ii}}-\mathrm{Ba} 1-\mathrm{F} 3^{\mathrm{iii}}$ & $74.9(6)$ & $\mathrm{F} 3^{\mathrm{i}}-\mathrm{Ba} 1-\mathrm{F} 4^{\mathrm{i}}$ & $52.0(4)$ \\
\hline $\mathrm{F} 3^{\mathrm{i}}-\mathrm{Ba} 1-\mathrm{F}^{\mathrm{iv}}$ & $74.9(6)$ & $\mathrm{F} 3^{\mathrm{ii}}-\mathrm{Ba} 1-\mathrm{F} 4^{\mathrm{i}}$ & $95.7(4)$ \\
\hline $\mathrm{F} 3^{\mathrm{ii}}-\mathrm{Ba} 1-\mathrm{F} 3^{\mathrm{iv}}$ & $129.1(3)$ & $\mathrm{F} 3^{\mathrm{iii}}-\mathrm{Ba} 1-\mathrm{F} 4^{\mathrm{i}}$ & $168.1(4)$ \\
\hline $\mathrm{F} 3^{\mathrm{iii}}-\mathrm{Ba} 1-\mathrm{F}^{\mathrm{iv}}$ & $129.1(3)$ & $\mathrm{F} 3^{\mathrm{iv}}-\mathrm{Ba} 1-\mathrm{F} 4^{\mathrm{i}}$ & $62.5(4)$ \\
\hline $\mathrm{F} 3^{\mathrm{i}}-\mathrm{Ba} 1-\mathrm{F} 1^{\mathrm{v}}$ & $105.4(4)$ & $\mathrm{F} 1^{\mathrm{v}}-\mathrm{Ba} 1-\mathrm{F} 4^{\mathrm{i}}$ & $54.6(4)$ \\
\hline 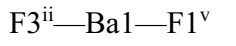 & $67.9(4)$ & $\mathrm{F} 1^{\mathrm{vi}}-\mathrm{Ba} 1-\mathrm{F} 4^{\mathrm{i}}$ & $128.6(4)$ \\
\hline 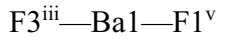 & $125.4(4)$ & $\mathrm{F} 1^{\mathrm{vii}}-\mathrm{Ba} 1-\mathrm{F} 4^{\mathrm{i}}$ & $63.2(4)$ \\
\hline $\mathrm{F} 3^{\mathrm{iv}}-\mathrm{Ba} 1-\mathrm{F} 1^{\mathrm{v}}$ & $62.1(4)$ & $\mathrm{F} 1-\mathrm{Ba} 1-\mathrm{F} 4^{\mathrm{i}}$ & $114.0(4)$ \\
\hline $\mathrm{F} 3^{\mathrm{i}}-\mathrm{Ba} 1-\mathrm{F} 1^{\mathrm{vi}}$ & $125.4(4)$ & $\mathrm{F} 4^{\mathrm{ii}}-\mathrm{Ba} 1-\mathrm{F} 4^{\mathrm{i}}$ & $117.4(3)$ \\
\hline $\mathrm{F} 3^{\mathrm{ii}}-\mathrm{Ba} 1-\mathrm{F} 1^{\mathrm{vi}}$ & $105.4(4)$ & $\mathrm{F} 4^{\mathrm{iv}}-\mathrm{Ba} 1-\mathrm{F} 4^{\mathrm{i}}$ & $94.6(6)$ \\
\hline $\mathrm{F} 3^{\mathrm{iii}}-\mathrm{Ba} 1-\mathrm{F} 1^{\mathrm{vi}}$ & $62.1(4)$ & $\mathrm{F} 4^{\mathrm{iii}}-\mathrm{Ba} 1-\mathrm{F} 4^{\mathrm{i}}$ & $117.4(3)$ \\
\hline $\mathrm{F} 3^{\mathrm{iv}}-\mathrm{Ba} 1-\mathrm{F}^{\mathrm{vi}}$ & $67.9(4)$ & $\mathrm{F} 2-\mathrm{Br} 1-\mathrm{F} 3$ & $92.6(6)$ \\
\hline $\mathrm{F} 1^{\mathrm{v}}-\mathrm{Ba} 1-\mathrm{F} 1^{\mathrm{vi}}$ & $90.93(7)$ & $\mathrm{F} 2-\mathrm{Br} 1-\mathrm{F} 4$ & $177.3(6)$ \\
\hline $\mathrm{F} 33^{\mathrm{i}}-\mathrm{Ba} 1-\mathrm{F} 1^{\mathrm{vii}}$ & $67.9(4)$ & $\mathrm{F} 3-\mathrm{Br} 1-\mathrm{F} 4$ & $84.9(6)$ \\
\hline $\mathrm{F} 3^{\mathrm{ii}}-\mathrm{Ba} 1-\mathrm{F} 1^{\mathrm{vii}}$ & $62.1(4)$ & $\mathrm{F} 2-\mathrm{Br} 1-\mathrm{F} 1$ & $92.9(6)$ \\
\hline $\mathrm{F} 3^{\mathrm{iii}}-\mathrm{Ba} 1-\mathrm{F} 1^{\mathrm{vii}}$ & $105.4(4)$ & $\mathrm{F} 3-\mathrm{Br} 1-\mathrm{F} 1$ & $174.4(6)$ \\
\hline $\mathrm{F} 3^{\mathrm{iv}}-\mathrm{Ba} 1-\mathrm{F} 1^{\mathrm{vii}}$ & $125.4(4)$ & $\mathrm{F} 4-\mathrm{Br} 1-\mathrm{F} 1$ & $89.6(6)$ \\
\hline $\mathrm{F} 1^{\mathrm{v}}-\mathrm{Ba} 1-\mathrm{F} 1^{\mathrm{vii}}$ & $90.93(7)$ & $\mathrm{Br} 1-\mathrm{F} 1-\mathrm{Ba} 1$ & $132.3(7)$ \\
\hline $\mathrm{F} 1^{\mathrm{vi}}-\mathrm{Ba} 1-\mathrm{F} 1^{\mathrm{vii}}$ & $165.4(5)$ & 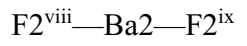 & $136.3(4)$ \\
\hline
\end{tabular}




\begin{tabular}{|c|c|}
\hline 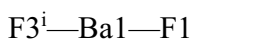 & $62.1(4)$ \\
\hline $\mathrm{F} 3^{\mathrm{ii}}-\mathrm{Ba} 1-\mathrm{F} 1$ & $125.4(4)$ \\
\hline 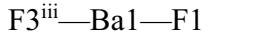 & $67.9(4)$ \\
\hline $\mathrm{F} 3^{\mathrm{iv}}-\mathrm{Ba} 1-\mathrm{F} 1$ & $105.4(4)$ \\
\hline $\mathrm{F} 1^{\mathrm{v}}-\mathrm{Ba} 1-\mathrm{F} 1$ & $165.4(5)$ \\
\hline $\mathrm{F} 1^{\mathrm{vi}}-\mathrm{Ba} 1-\mathrm{F} 1$ & $90.93(7)$ \\
\hline 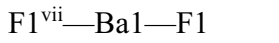 & $90.93(7)$ \\
\hline $\mathrm{F} 3^{\mathrm{i}}-\mathrm{Ba} 1-\mathrm{F} 4^{\mathrm{ii}}$ & $168.1(4)$ \\
\hline $\mathrm{F} 3^{\mathrm{ii}}-\mathrm{Ba} 1-\mathrm{F} 4^{\mathrm{ii}}$ & $52.0(4)$ \\
\hline 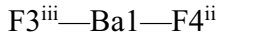 & $62.5(4)$ \\
\hline $\mathrm{F} 3^{\mathrm{iv}}-\mathrm{Ba} 1-\mathrm{F} 4^{\mathrm{ii}}$ & 95.7 (4) \\
\hline $\mathrm{F} 1^{\mathrm{v}}-\mathrm{Ba} 1-\mathrm{F} 4^{\mathrm{ii}}$ & $63.2(4)$ \\
\hline 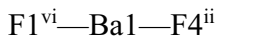 & $54.6(4)$ \\
\hline 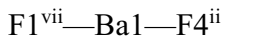 & $114.0(4)$ \\
\hline $\mathrm{F} 1-\mathrm{Ba} 1-\mathrm{F} 4^{\mathrm{ii}}$ & $128.6(4)$ \\
\hline $\mathrm{F}^{\mathrm{i}}-\mathrm{Ba} 1-\mathrm{F}^{\mathrm{iv}}$ & $62.5(4)$ \\
\hline $\mathrm{F} 3^{\mathrm{ii}}-\mathrm{Ba} 1-\mathrm{F} 4^{\mathrm{iv}}$ & $168.1(4)$ \\
\hline 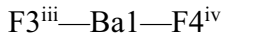 & $95.7(4)$ \\
\hline $\mathrm{F} 3^{\mathrm{iv}}-\mathrm{Ba} 1-\mathrm{F} 4^{\mathrm{iv}}$ & $52.0(4)$ \\
\hline $\mathrm{F} 1^{\mathrm{v}}-\mathrm{Ba} 1-\mathrm{F} 4^{\mathrm{iv}}$ & $114.0(4)$ \\
\hline 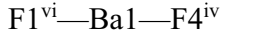 & $63.2(4)$ \\
\hline $\mathrm{F} 1^{\mathrm{vii}-\mathrm{Ba} 1-\mathrm{F} 4^{\mathrm{iv}}}$ & $128.6(4)$ \\
\hline $\mathrm{F} 1-\mathrm{Ba} 1-\mathrm{F} 4^{\mathrm{iv}}$ & $54.6(4)$ \\
\hline 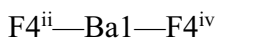 & $117.4(3)$ \\
\hline $\mathrm{F} 3^{\mathrm{i}}-\mathrm{Ba} 1-\mathrm{F}^{\mathrm{iii}}$ & $95.7(4)$ \\
\hline 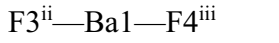 & $62.5(4)$ \\
\hline 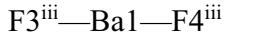 & $52.0(4)$ \\
\hline $\mathrm{F} 3^{\mathrm{iv}}-\mathrm{Ba} 1-\mathrm{F} 4^{\mathrm{iii}}$ & $168.1(4)$ \\
\hline $\mathrm{F} 1^{\mathrm{v}}-\mathrm{Ba} 1-\mathrm{F} 4^{\mathrm{iii}}$ & $128.6(4)$ \\
\hline $\mathrm{F} 1^{\mathrm{vi}}-\mathrm{Ba} 1-\mathrm{F} 4^{\mathrm{iii}}$ & $114.0(4)$ \\
\hline 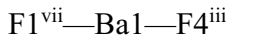 & $54.6(4)$ \\
\hline $\mathrm{F} 1-\mathrm{Ba} 1-\mathrm{F} 4^{\mathrm{iii}}$ & $63.2(4)$ \\
\hline $\mathrm{F} 3-\mathrm{Br} 1-\mathrm{F} 2-\mathrm{Ba} 2$ & $103.8(13)$ \\
\hline $\mathrm{F} 1-\mathrm{Br} 1-\mathrm{F} 2-\mathrm{Ba} 2$ & $-75.4(13)$ \\
\hline
\end{tabular}

\begin{tabular}{|c|c|}
\hline $\mathrm{F} 2^{\mathrm{viii}}-\mathrm{Ba} 2-\mathrm{F} 2^{\mathrm{x}}$ & $63.5(6)$ \\
\hline $\mathrm{F} 2^{\mathrm{ix}}-\mathrm{Ba} 2-\mathrm{F} 2^{\mathrm{x}}$ & $136.3(4)$ \\
\hline $\mathrm{F} 2^{\mathrm{viii}}-\mathrm{Ba} 2-\mathrm{F} 2$ & $136.3(4)$ \\
\hline $\mathrm{F} 2^{\mathrm{ix}}-\mathrm{Ba} 2-\mathrm{F} 2$ & $63.5(6)$ \\
\hline $\mathrm{F} 2^{\mathrm{x}}-\mathrm{Ba} 2-\mathrm{F} 2$ & $136.3(4)$ \\
\hline 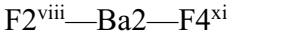 & $114.0(5)$ \\
\hline $\mathrm{F} 2^{\mathrm{ix}}-\mathrm{Ba} 2-\mathrm{F} 4^{\mathrm{xi}}$ & $109.7(5)$ \\
\hline $\mathrm{F} 2^{\mathrm{x}}-\mathrm{Ba} 2-\mathrm{F} 4^{\mathrm{xi}}$ & $67.9(5)$ \\
\hline $\mathrm{F} 2-\mathrm{Ba} 2-\mathrm{F} 4^{\mathrm{xi}}$ & $68.4(5)$ \\
\hline $\mathrm{F}^{\mathrm{viii}}-\mathrm{Ba} 2-\mathrm{F} 4^{\mathrm{vii}}$ & $67.9(5)$ \\
\hline $\mathrm{F} 2^{\mathrm{ix}}-\mathrm{Ba} 2-\mathrm{F} 4^{\mathrm{vii}}$ & $68.4(5)$ \\
\hline $\mathrm{F} 2^{\mathrm{x}}-\mathrm{Ba} 2-\mathrm{F} 4^{\mathrm{vii}}$ & $114.0(5)$ \\
\hline $\mathrm{F} 2-\mathrm{Ba} 2-\mathrm{F} 4^{\mathrm{vii}}$ & $109.7(5)$ \\
\hline $\mathrm{F} 4^{\mathrm{xi}}-\mathrm{Ba} 2-\mathrm{F}^{\mathrm{vii}}$ & $178.0(7)$ \\
\hline 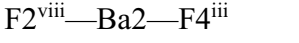 & $68.4(5)$ \\
\hline $\mathrm{F} 2^{\mathrm{ix}}-\mathrm{Ba} 2-\mathrm{F} 4^{\mathrm{iii}}$ & $114.0(5)$ \\
\hline $\mathrm{F} 2^{\mathrm{x}}-\mathrm{Ba} 2-\mathrm{F} 4^{\mathrm{iii}}$ & $109.7(5)$ \\
\hline $\mathrm{F} 2-\mathrm{Ba} 2-\mathrm{F} 4^{\mathrm{iii}}$ & $67.9(5)$ \\
\hline $\mathrm{F} 4^{\mathrm{xi}}-\mathrm{Ba} 2-\mathrm{F} 4^{\mathrm{iii}}$ & $90.018(16)$ \\
\hline 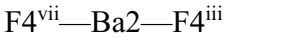 & $90.018(15)$ \\
\hline $\mathrm{F} 2^{\mathrm{viii}-\mathrm{Ba} 2-\mathrm{F} 4^{\mathrm{xii}}}$ & $109.7(5)$ \\
\hline $\mathrm{F} 2^{\mathrm{ix}}-\mathrm{Ba} 2-\mathrm{F} 4^{\mathrm{xii}}$ & $67.9(5)$ \\
\hline $\mathrm{F} 2^{\mathrm{x}}-\mathrm{Ba} 2-\mathrm{F} 4^{\mathrm{xii}}$ & $68.4(5)$ \\
\hline $\mathrm{F} 2-\mathrm{Ba} 2-\mathrm{F} 4^{\mathrm{xii}}$ & $114.0(5)$ \\
\hline $\mathrm{F} 4^{\mathrm{xi}}-\mathrm{Ba} 2-\mathrm{F} 4^{\mathrm{xii}}$ & $90.018(15)$ \\
\hline $\mathrm{F} 4^{\mathrm{vii}-\mathrm{Ba} 2-\mathrm{F} 4^{\mathrm{xii}}}$ & $90.018(15)$ \\
\hline $\mathrm{F} 4^{4 i i}-\mathrm{Ba} 2-F 4^{\mathrm{xii}}$ & $178.0(7)$ \\
\hline $\mathrm{Br} 1-\mathrm{F} 2-\mathrm{Ba} 2$ & $146.6(8)$ \\
\hline $\mathrm{Br} 1-\mathrm{F} 3-\mathrm{Ba} 1^{\mathrm{xiii}}$ & $114.9(6)$ \\
\hline $\mathrm{Br} 1-\mathrm{F} 4-\mathrm{Ba} 2^{\mathrm{xiv}}$ & $120.5(6)$ \\
\hline $\mathrm{Br} 1-\mathrm{F} 4-\mathrm{Ba} 1^{\mathrm{xii}}$ & $103.2(5)$ \\
\hline $\mathrm{Ba} 2^{\mathrm{xiv}}-\mathrm{F} 4-\mathrm{Ba} 1^{\mathrm{xiii}}$ & $130.1(5)$ \\
\hline $\mathrm{F} 2-\mathrm{Br} 1-\mathrm{F} 3-\mathrm{Ba} 1^{\mathrm{xiii}}$ & $158.2(7)$ \\
\hline $\mathrm{F} 4-\mathrm{Br} 1-\mathrm{F} 3-\mathrm{Ba} 1^{\mathrm{xiii}}$ & $-20.9(7)$ \\
\hline
\end{tabular}

Symmetry codes: (i) $y-1 / 2,-x+3 / 2,-z+3 / 2$; (ii) $x-1 / 2, y-1 / 2, z-1 / 2$; (iii) $-x+3 / 2,-y+3 / 2, z-1 / 2$; (iv) $-y+3 / 2, x-1 / 2,-z+3 / 2$; (v) $-x+1,-y+1, z$; (vi) $y$, $-x+1,-z+1$; (vii) $-y+1, x,-z+1$; (viii) $y-1 / 2,-x+3 / 2,-z+1 / 2$; (ix) $-x+1,-y+2, z$; (x) $-y+3 / 2, x+1 / 2,-z+1 / 2$; (xi) $y,-x+2,-z+1$; (xii) $x-1 / 2, y+1 / 2, z-1 / 2$; (xiii) $x+1 / 2, y+1 / 2, z+1 / 2$; (xiv) $x+1 / 2, y-1 / 2, z+1 / 2$. 